\title{
The Guidance Layer of the Cul-de-sac Courtyard Physical Environment Evaluation Toolkit (CPEET)
}

\author{
Anniz Fazli Ibrahim Bajunid a, Mohamed Yusoff Abbasa, \\ Abdul Hadi Nawawi ${ }^{\text {b }}$, Wan Norhishamuddin Wan Rodi ${ }^{b}$ \\ a Centre for Environment-Behaviour Studies \\ ${ }^{b}$ Department of Estate Management \\ Faculty of Architecture, Planning and Surveying, \\ Universiti Teknologi MARA (UiTM), Shah Alam, Malaysia \\ toanniz@gmail.com
}

\begin{abstract}
The environment-behaviour of a cul-de-sac courtyard poses an array of intriguing questions, both qualitatively and quantitatively. In establishing a systematic and reliable evaluation of these microneighbourhoods, a sequence of procedural best practices of an evaluation toolkit was introduced. Documenting measurable evidences as well as illustrating the anecdotal nature of a cul-de-sac courtyard requires specific and duplicable chronicling processes. The Cul-de-sac Courtyard Physical Environment Evaluation Toolkit (CPEET), adapted appropriately from AEDET Evolution and ASPECT, became the foundation of the toolkit.
\end{abstract}

Keywords: Cul-de-sac courtyards; evaluation toolkit; best practice; cul-de-sac syntax

eISSN: 2398-4279 @ 2016. The Authors. Published for AMER ABRA by e-International Publishing House, Ltd., UK.. This is an open access article under the CC BY-NC-ND license (http://creativecommons.org/licenses/by-ncnd/4.0/). Peer-review under responsibility of AMER (Association of Malaysian Environment-Behaviour Researchers), ABRA (Association of Behavioural Researchers on Asians) and cE-Bs (Centre for EnvironmentBehaviour Studies), Faculty of Architecture, Planning \& Surveying, Universiti Teknologi MARA, Malaysia.

https://doi.org/10.21834/ajqol.v1i1.32 


\subsection{Introduction}

In ascertaining the quality of life, especially within the micro-neighbourhood environment is an extensive task for designers, architects and planners. More often than not, these environment-behaviour patterns are widely discussed in western societies rather than that of developing nations. The residential typology of the cul-de-sac courtyard remains foreign to authorities but has, in advanced nations, offered heated debates of profound consequences. The Southeast Asian context of Malaysia as well needs much examination due to the contextual differences and complexities of numerous scientific domains.

The AEDET Evolution (Achieving Excellence Design Evaluation Toolkit) and ASPECT (A Staff/Patient Environment Calibration Toolkit) by the Department of Health in the United Kingdom, forms the main basis of the papers' toolkit adoption (guidance layer). Locally, there have been attempts by researchers and local authorities such as Federal Department of Town and Country Planning (2013), Mohit \& Elsawahli (2010) and Othman \& Said (2012). The local researchers' contributions are very promising, but will require time to evolve and mature. This paper also projects to contribute to that pool of knowledge.The objective of this paper is to disclose best practices of procedures and the documentation of data of CPEET (Cul-de-sac Courtyard Physical Environment Evaluation Toolkit), during the collection and reporting processes. This paper shall strive to elucidate these best practices and illustrate contextual examples pertaining to cul-de-sac courtyards in the Shah Alam municipality. The examples provided shall exhibit the effectiveness of these best practices in identifying both the quantitative and qualitative elements of the research undertaking.

The paper shall be divided into three (3) sections. The first initiating the discussion on AEDET Evolution and ASPECT. The second shall rationalise the design and implementation of CPEET itself. The final section discusses CPEETs' collected data samples, with reference for further potential.

\subsection{Literature Review}

\subsection{Discourses on procedures and documenting data}

Relevant Precedents - AEDET Evolution \& ASPECT

AEDET Evolution (Achieving Excellence Design Evaluation Toolkit) by the Department of Health in the United Kingdom has over 1000 growing body of scientific research using its toolkit (DH Estates \& Facilities, 2007).

The AEDET Evolution \& ASPECT toolkits arise from the need to evaluate the multifaceted issues of healthcare facilities, which are difficult to measure or evaluate. The toolkit was designed to simplify this process by establishing clear and non-technical scoring statements around three (3) vital areas of design and healthcare (Impact, Build Quality and Functionality). It is widely used in the United Kingdoms' Trusts and the NHS (DH Estates \& Facilities, 2012a). 
ASPECT on the other hand, has over 600 expanding number of research within its database (DH Estates \& Facilities, 2012b). It can be used as a standalone toolkit or in support of AEDET Evolution. However, ASPECT primarily focuses on the environmental impact of healthcare on satisfaction of staff (performance) and patients (health outcomes). ASPECT concentrates on eight (8) major themes (Privacy, company \& dignity, Views, Nature \& outdoors, Comfort \& control, Legibility of place, Interior appearance, Facilities and Staff).

The above two (2) toolkits comprises of three (3) mechanisms or layers. These layers are also typically best practices and guidelines for the conduct of the research. The Guidance layer describes the themes / factors in detail, the Scoring layer typically assists the scoring processes, whilst the Evidence layer directs to research evidences. This papers' Cul-de-sac Courtyard Physical Environment Evaluation Toolkit (CPEET) comprises of both AEDET Evolution and ASPECTS fundamental approach. The justification behind the combination of both these toolkit is fairly logical and realistic in the application of the external micro-neighbourhood physical environment. Wherein, the externally designed physical environments are multifaceted and are difficult to measure. Whilst the environmental impact on residents' behaviour and satisfaction are the primary focus.

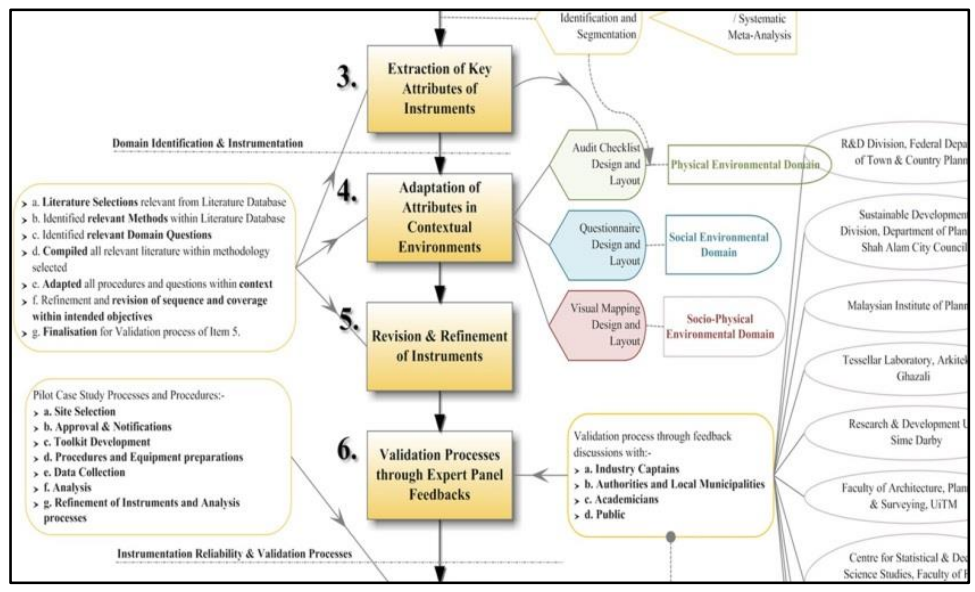

Figure 1: A snapshot of a portion of the refinement flowchart and processes involved, from left to right (Author). For a complete and detailed description on the figure, please contact the Author.

The process of contextualising these adaptations required over 11 revisions. Extraction, adaptation and refinement of the toolkit required the input from relevant experts, industry captains and authorities (Figure 1). Figure 1 typically delineates the processes of the refinement flowchart in ensuring the reliability and validity of the toolkit. Figure 1 also took 
into consideration the numerous critical characteristics or themes of both AEDET Evolution and ASPECT.

\subsection{Methodology}

\subsection{Toolkit design and implementation}

\subsubsection{Adapting AEDET \& ASPECT into CPEET}

The essence of CPEET reflects that of AEDET Evolution and ASPECT from two (2) areas of integration, namely the Guidance layer and the Scoring layer. The Guidance layer (the basis of this papers' focus) or Best Practices was primarily adopted from AEDETs' and ASPECTs' Information Reader Box (DH Estates \& Facilities, 2012a,b).

\subsubsection{Subsections of the CPEET guidance layer / instructional design}

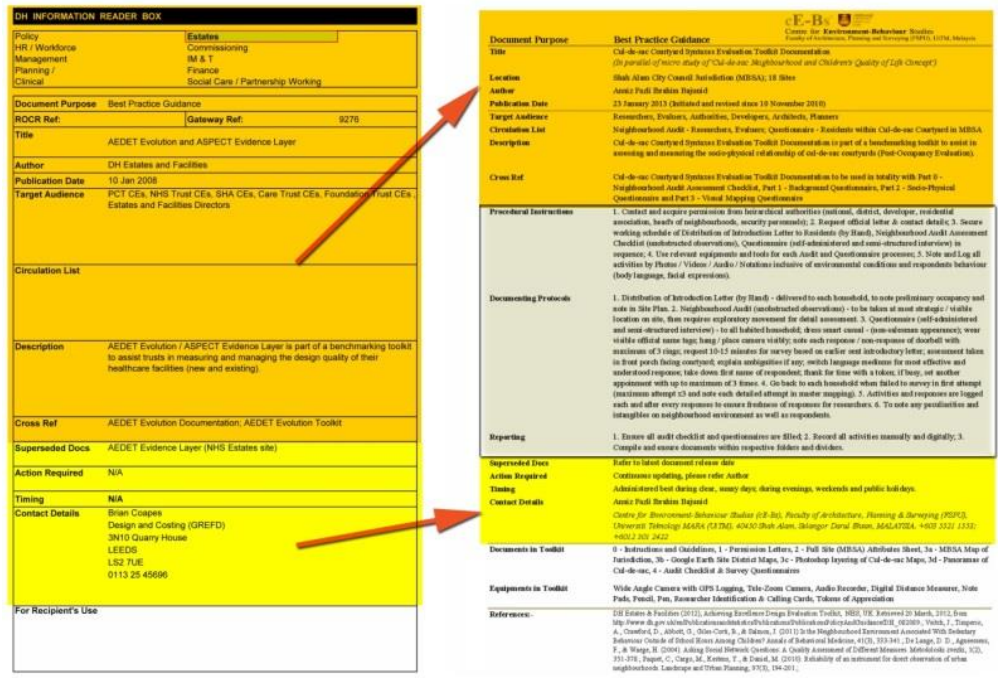

Figure 2: Best Practice Guidance Layer of CPEET (Author). The illustration depicts the different sections of the guidance layer / instructional design of CPEET (right), adopted from AEDET Evolution and ASPECT (left). CPEET details the documents intentions, researchers' instructions, requirements as well as tools and equipment involved explicitly.

CPEET consists of six (6) sections (Figure 2). The first section comprises of relevant details pertaining to the particular site, i.e. title, location, author and publication date. The second section details the target audience, circulation list and a brief description of the toolkit. 
Whereas the last two (2) sections, consists of important information such as superseding documents, actions required, time proposed, contact details of researcher, toolkit documents, equipment and further references of the toolkit. Section three (3) consisting of cross-referencing, procedures, documenting protocols and reporting will be discussed next in detail for a more thorough understanding of the toolkit. All these sections are in tandem to AEDET Evolution and ASPECT and could be seen in the illustrated Figure 2.

\subsubsection{Cross referencing}

CPEET Documentation is to be used in totality with the following documents. This is to ascertain holistic appreciation of data collected for a particular cul-de-sac neighbourhood.

- $\quad$ Part 0 - Neighbourhood Audit Assessment Checklist (Unobstructed Observations)

- $\quad$ Part 1 - Background Questionnaire (Researcher-administered)

- $\quad$ Part 2 - Socio-Physical Questionnaire (Researcher-administered)

- $\quad$ Part 3 - Visual Mapping Questionnaire (Semi-structured interview)

\subsubsection{Procedural instructions}

The procedures involved in administering the toolkit effectively encompasses the following processes, (but are not limited to, due to site specific context):-

- $\quad$ Contact, acquire and record all permissions and contact details of authorities / person-in-charge in a hierarchical manner (national, district, residential association and or developer, neighbourhoods' chair / head and head of security personnel, for gated and guarded neighbourhoods).

- $\quad$ Researcher to request an official letter of approval / consent with relevant contact details, (where possible from all persons in charge).

- $\quad$ Secure visit / working schedule of:- i - Distribution of Introductory Letter to Residents (with stamped organisation address and delivered By Hand); ii Neighbourhood Audit Assessment Checklist (unobstructed observations); iii Questionnaire (researcher-administered and semi-structured interview) in sequence.

- Use relevant equipment and tools for each Audit and Questionnaire processes.

- Note and Log all activities by Photos / Videos / Audios / Notations, inclusive of environmental conditions and respondents behaviour (body language, facial expressions), when conditions necessitates.

\subsubsection{Documenting protocols}

The protocols in documenting data are of the essence in ensuring best practices are met by adhering to the following:-

- Distribution of the Introductory Letter - delivered to each household, in three (3) stages (three months prior to survey; when embarking within neighbourhood Seksyen; and a week prior to specific neighbourhood survey). 
- Researchers are to note all occupied residences on the Site Plan during delivery of the introductory letters.

- $\quad$ Part 0 - Neighbourhood Audit - to be observed by researcher at the most strategic / visible location on site but will then require constant movement for detail assessment of each of the audit checklist.

- $\quad$ Part 1-3 Questionnaire (researcher-administered and semi-structured interview) - to all occupied households. Researchers are to be smartly dressed (smart-casual non-salesman appearance) and wear official nametags with camera (visible).

- $\quad$ Researcher then to note each house's 'doorbell' response (or non-response) and to limit ringing of 'doorbell' to maximum of three (3) rings.

- $\quad$ Researcher to request politely from respondents 10-15 minutes of their time for survey (to refer to sent introductory letter). Questionnaire to be conducted on the front porch, facing the courtyard, where relevance of each question is shown best in the context. Researchers to translate / switch language medium for effective and understood response for any ambiguities. Researcher finally to take note each respondent's first name for research management records (to clarify with respondents' anonymity).

- Researcher to ensure finally, that each respondent is thanked for his or her time and to present him or her, a token of appreciation (the goodie bag).

- If respondent busy, set up an appointment with up to maximum of three (3) rescheduled appointments.

- $\quad$ Researcher to return to each house when unable to survey on the first attempt due to non-response (maximum of three (3) attempts) and to record each attempts' date and time in master mapping.

- Activities and responses are logged each and after every completed questionnaire, to ensure 'freshness' of comments (whenever possible).

- Researcher to note any peculiarities and intangible behaviours of respondents or of their neighbourhood environment.

\subsubsection{Reporting}

Best practices for reporting the data includes:-

- Ensuring all audit checklist and questionnaires are filled.

- Notations of all research activities to be manually and digitally recorded in hard and soft copies.

- $\quad$ Researchers to compile and ensure each respective document is sorted within its respective folders and dividers of management file.

\subsection{Implementing the toolkit}

The evaluation toolkit functions as the primary tool in obtaining and subsequently documenting the data. In this particular instance, the toolkit underwent strict trials in a pilot survey of two (2) cul-de-sac neighbourhoods of Seksyen 35. Subsequent to the survey 
conducted, the toolkit was further refined an additional 11 times as discussed earlier. This refinement includes the reliability and validity of the questionnaires itself, apart from the general outlines of best practices of administering the toolkit. These processes were recorded in further detail by Bajunid, et. al. (2013a, b), where relevant academic, industry and authority experts were consulted.

\subsection{Toolkit reporting}

Data management of the various data collected is crucial. Thus, the reporting template (along with other supporting documents) functions as an executive summary or a point of first reference. The reporting template consists of three (3) main sections of map demarcation, panorama and pictures (with audio and video links) and an executive summary (Figure. 3). The first two (2) sections contain quantitative physical data collected on site whereas the third section is an anecdote (field notes) from the researchers experience and observations from their multiple visits and interactions with the respondents in each site. This section is rather noteworthy where the subtleties of behaviours are recorded for further discussion.

\subsection{Results and Discussions}

\subsection{Executive summary \& reporting}

The particular study on the cul-de-sac courtyard and the environment behaviour of its occupants are a sizeable. Shah Alam municipality has within its neighbourhood typology of the cul-de-sac courtyard, 18 sites. This can be further stratified into six (6) neighbourhoods to grasp the nuances or syntaxes of each (Bajunid, et. al. 2012, 2013c).

Cross referencing the executive summaries of researchers (observational) and residents responses (through questionnaires). Table 1 summarises the qualitative anecdotal summaries of the researchers within the six (6) neighbourhoods. What is stimulating to observe is that the quantitative results of the questionnaires (also shown within table) displayed corresponding values to that of the summaries. It would be fascinating to delve into data interpretation; this paper only focuses on the effectiveness of the toolkit. It is however, important to note that, whilst the table represents only a simple summary, the data was streamlined from 18 different cul-de-sac courtyard sites, with at least 1242 household visits of 414 available houses for the survey of which 166 residents responded (Bajunid, et. al. 2013c) to 49 items from 10 subscales (Bajunid, et. al. 2014) in a census survey. As explained earlier, the procedures and data management of the toolkit has allowed for a vast amount of quantitative and qualitative data to be collected, recorded and streamlined. The validity and reliability of this toolkit has begun to manifest within Table 1. Nevertheless, additional thorough analysis is clearly required sequentially to this paper. 


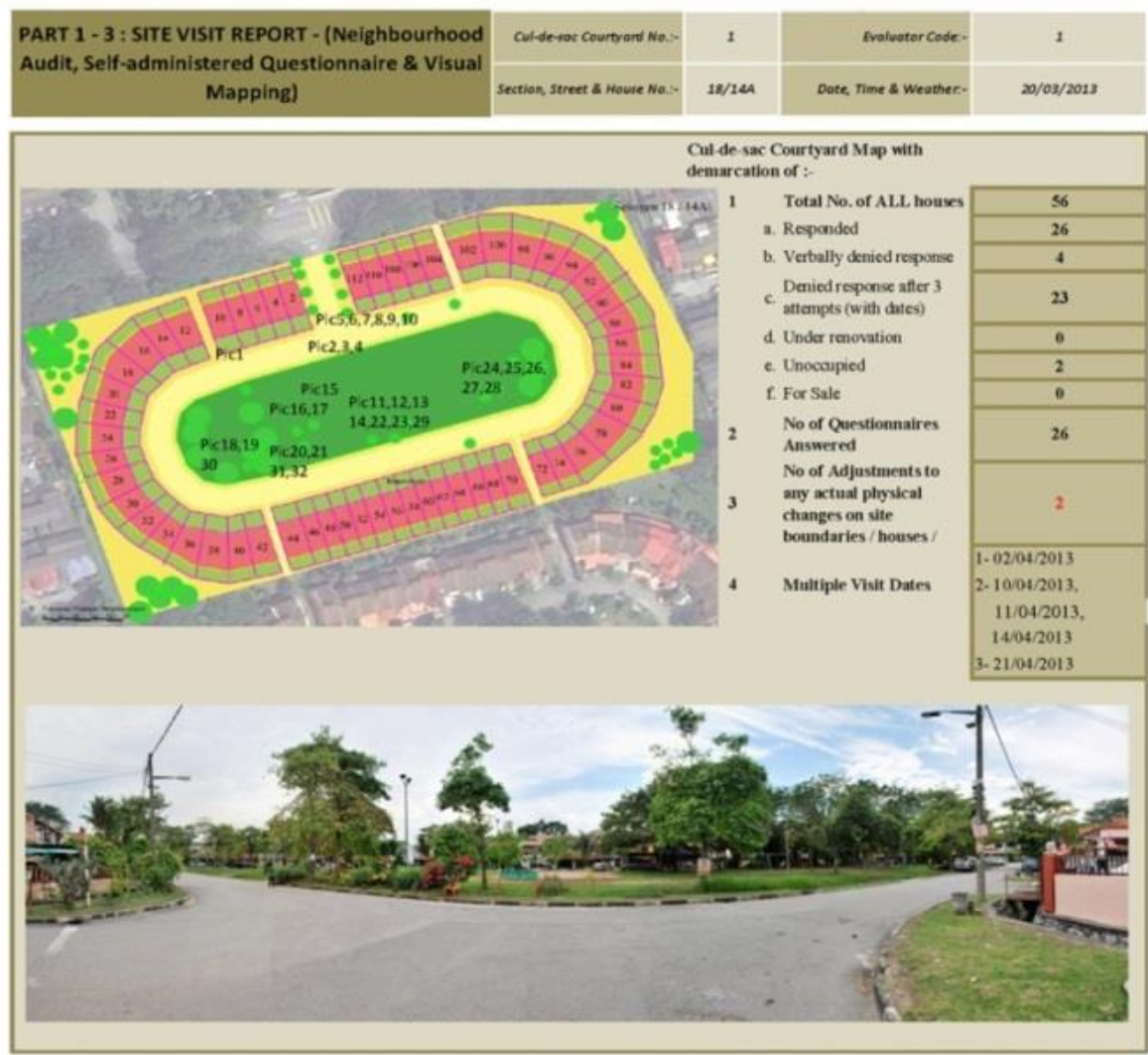

Figure 3: Illustration portrays a reporting sample of a neighbourhood and its qualitative and quantitative data, after each successful collection of all data on the site, has been made (portion of an extensive reporting template. More information could be obtained from Author). Three (3) sections of mapping, pictorial records (also with audio and video links) and anecdotal summary, can be seen within the executive summary report. The full dossier of each cul-de-sac courtyard neighbourhood however, is not shown. 


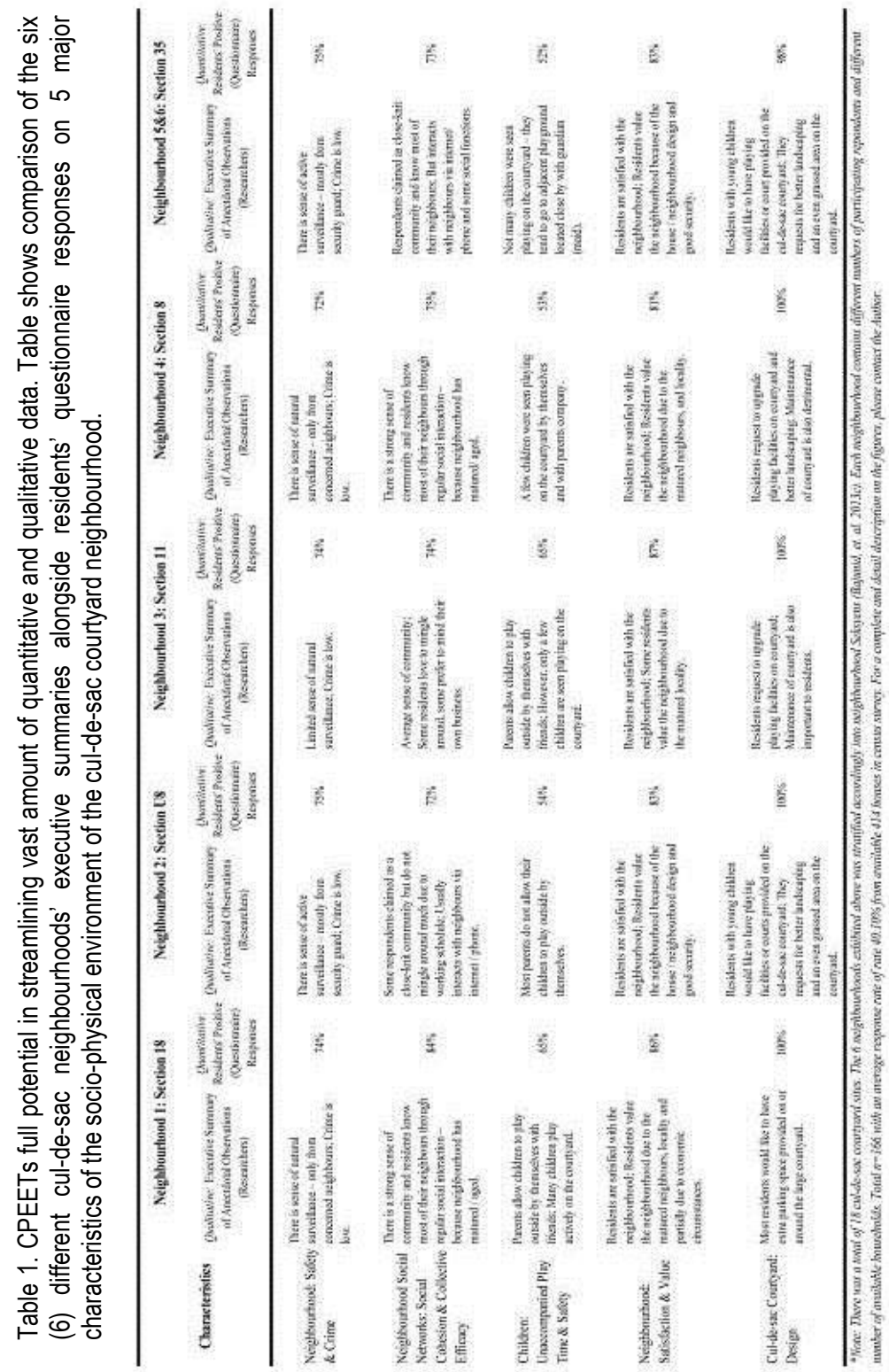




\subsection{Conclusion}

The paper intended to divulge the best practices of procedures and the documentation of data during the collection and reporting processes through the usage of CPEET that may lead to an effective research undertaking. Though successful to a point, several issues can still be addressed to crystallise further its approach and reduce ambiguities.

It is not yet known of the extent of CPEETs' limitations and parameters. Whilst the best practices seems credible within the context of a micro-neighbourhood environment, its applications within different settings and scales remains to be seen. The foundational basis of its Guidance layer adopted (in context) from AEDET Evolution and ASPECT has in a way, propelled the toolkits potential, providing the CPEET convincing avenues for further development. The continuance of CPEET's usage shall also allow for the development of the Evidence layer, where a catalogued database will encourage further scientific discussions amongst practitioners and researchers.

Few scholastic toolkit of the external environment of micro-neighbourhoods in Malaysia demonstrates original research but requires numerous controlling considerations. CPEET's adaptation, differing from its original adoption, requires further stringent reliability and validity assessments. However, as discussed, the controlled census survey in this paper within the managing of data's Guidance layer, demonstrated convincing validity and reliability. CPEET's best practices can be immediately used and continuously fine-tuned to case specific research, constantly building its own Evidence layer.

In the absence of local scientific database for CPEET's usage, triangulations of data will substantiate and strengthen its findings. CPEET will have to undergo different scientific adoptions within the context of the external physical environment to be able to validate its robustness.

In essence, CPEET's instructional designs or guidance layer, along with examples of its resulting procedures and processes have been expressed explicitly. It is anticipated that the adoption of this toolkit will continue to expand in fulfilling other research initiatives. It is also hoped by distilling each procedure and processes of the toolkit, the fundamental crux of the expanding discovery of quality of life within micro-neighbourhoods, could be better understood.

\section{Acknowledgement}

The Author is appreciative to the supervisory committee and industry collaborators for their practical comments. The Authors commends the dedicated work of Graduate Research Assistant, Syazwani Abdul Kadir made possible by the Research Acculturation Grant Scheme (RAGS) by the Ministry of Higher Education and Universiti Teknologi MARA (UiTM). The Author wishes to also acknowledge the complete and extended version of this article which can be sourced from Procedia - Social and Behavioural Sciences. 


\section{References}

Bajunid, A. F. I., Abbas, M. Y., \& Nawawi, A. H. (2012). Identification processes of culs-de-sac in Shah Alam: Preamble to neighbourhoods' way of life. Procedia Social and Behavioral Sciences, 50 (2012), 226-237. Doi: 10.1016/j.sbspro.2012.08.030.

Bajunid, A. F. I., Abbas, M. Y., \& Nawawi, A. H. (2013a). Assessing cul-de-sac neighbourhoods: A methodological prelude. Procedia Social and Behavioral Sciences, 84(2013), 288-292. Doi: 10.1016/j.sbspro.2013.06.553.

Bajunid, A. F. I., Abbas, M. Y., Nawawi, A. H., Iyer, M.K.S.G., Rodi, W.N.W. \& Azhari, N.F.N. (2014). Questionnaires in review: Socio-mapping the cul-de-sac courtyards of Shah Alam. Procedia Social and Behavioral Sciences, 114(2014), 559-563. Doi: 10.1016/j.sbspro.2013.12.747.

Bajunid, A. F. I., Abbas, M. Y., \& Nawawi, A. H. (2013c). Demystifying the cul-de-sac courtyards syntaxes. Procedia Social and Behavioral Sciences, 105(2013), 525-535. doi: 10.1016/j.sbspro.2013.11.056.

DH Estates \& Facilities (2012a). Achieving excellence design evaluation toolkit (AEDET), NHS, UK. Retrieved 20 March, 2012, from http://www.dh.gov.uk/en/Publicationsandstatistics/Publications/PublicationsPolicyAndGuidance/DH_082089

DH Estates \& Facilities (2012b). A staff and patient environment calibration toolkit (ASPECT), NHS, UK. Retrieved 20 March, 2012, from http://www.dh.gov.uk/en/Publicationsandstatistics/Publications/PublicationsPolicyAndGuidance/DH_082087

DH Estates \& Facilities. (2007). R\&D Project B(04)03: Disseminating Good Practice (GDP): developing an exemplar layer for AEDET Evolution and ASPECT design evaluation tools, NHS, Wales. Retrieved 20 October, 2013, from http://www.wales.nhs.uk/sites3/page.cfm?orgid=254\&pid=7615.

Federal Department of Town and Country Planning (2013). Garis Panduan Perancangan Perumahan (Draf Ketujuh 4 Januari 2013). (ISBN 978-983-41729-6-1). Malaysia: Federal Department of Town and Country Planning, Ministry of Housing and Local Government Malaysia.

Mohit, M. A., \& Elsawahli, H. M. H. (2010). Crime and Housing in Malaysia: Case Study of Taman Melati Terrace Housing in Kuala Lumpur. Asian Journal of Environment-Behaviour Studies, 1(3), 25-36.

Othman, S., \& Said, I. (2012). Affordances of Cul-de-sac in Urban Neighborhoods as Play Spaces for Middle Childhood Children. Procedia - Social and Behavioral Sciences, 38(0), 184-194. Doi: 10.1016/j.sbsp ro.2012.03.339. 